\title{
Le maître lasallien en Bulgarie : entre le vœu et la réalité sociopolitique et culturelle bulgare
}

The Lasallian teacher in Bulgaria: between the wish and Bulgarian socio-

political and cultural reality

Julieta Velichkova-Borin

\section{OpenEdition}

Édition électronique

URL : https://journals.openedition.org/dhfles/8466

DOI : $10.4000 /$ dhfles.8466

ISSN : 2221-4038

Éditeur

Société Internationale pour l'Histoire du Français Langue Étrangère ou Seconde

Édition imprimée

Date de publication : 1 décembre 2021

ISSN : $0992-7654$

Référence électronique

Julieta Velichkova-Borin, « Le maître lasallien en Bulgarie : entre le vœu et la réalité sociopolitique et culturelle bulgare », Documents pour l'histoire du français langue étrangère ou seconde [En ligne], 66-67| 2021, mis en ligne le 03 février 2022, consulté le 03 avril 2023. URL : http://journals.openedition.org/ dhfles/8466 ; DOI : https://doi.org/10.4000/dhfles.8466

Ce document a été généré automatiquement le 3 avril 2023.

Tous droits réservés 


\section{Le maître lasallien en Bulgarie : entre le vœu et la réalité sociopolitique et culturelle bulgare ${ }^{1}$}

The Lasallian teacher in Bulgaria: between the wish and Bulgarian sociopolitical and cultural reality

Julieta Velichkova-Borin

D'après Max Roche (1989), qui essaie d'éclaircir la motivation des frères en Orient, en se basant sur un texte du missionnaire et enseignant au collège des lazaristes à Constantinople, Eugène Boré, on peut penser que les frères viennent avec l'espoir de convertir les chrétiens des autres rites, ainsi que les musulmans. Cependant, selon les observations de Paul Imbert (1909), au Levant la conversion religieuse semble impossible. Karène Sanchez-Summerer (2015) souligne la participation des frères à la mise en place de la didactique du français en tant que langue étrangère, sans pourtant oublier que "officiellement leur but premier est resté l'évangélisation» (Ibid. : 97). Notons à ce propos que les cas de conversion, dans les trois écoles lasalliennes en Bulgarie sont plutôt rares. Les résultats et les effets du travail et de la présence des frères en Bulgarie demeurent en partie méconnus, car ils sont observables et appréciables, entre autres, à travers l'activité de leurs élèves qui, dans la plupart des cas, évitent de parler de leur formation dans les écoles catholiques françaises pendant la période qui suit leur fermeture en Bulgarie, en 1948, pour des raisons d'ordre politique. Selon un diplomate français, cité par Raïa Zaïmova (2007: 152), « c'est parmi les anciens élèves que la jeune Bulgarie avait recruté ses premiers fonctionnaires ».

Il semble utile de réfléchir sur la sélection et la formation des frères, formés dans la plupart des cas au noviciat de Saint-Maurice l'Exil (Isère) ; sur les principes et les acquis pédagogiques de l'École lasallienne dans une perspective historique plus large ; sur les méthodes d'enseignement $\mathrm{du} / \mathrm{en}$ français langue étrangère, appliquées dans les écoles lasalliennes en Bulgarie dans un milieu sociopolitique et culturel spécifique présupposé être plutôt hostile, inégal et mouvementé, compte tenu des circonstances. 
3 Notre texte sera focalisé sur les valeurs et les activités de ces «ouvriers, qui restent dans l'ombre et sont pourtant, par leur action quotidienne sur le terrain et dans la durée, les artisans de la réussite » (Michel $2004: 41$ ) des écoles catholiques françaises de Sofia et de Roussé5.

\section{Le statut des écoles catholiques françaises en Bulgarie (1864-1948)}

4 La motivation de l'ouverture de ces écoles est d'ordre religieux. Elle est directement saisissable sur le registre missionnaire catholique: "destruction du schisme " et « retour à la foi des populations orientales ».

5 Ces écoles relèvent de deux vecteurs de première importance dans l'espace socioculturel français et francophone : la politique linguistique et le catholicisme. La langue française, en tant que lingua franca, langue véhiculaire pour les relations commerciales, mais aussi diplomatiques et culturelles, semble encore sans égale au $\mathrm{XIX}^{\mathrm{e}}$ siècle. La France, en tant que puissance catholique qui s'est imposée comme protectrice des chrétiens dans l'Empire ottoman, suscite toujours la confiance des Bulgares.

6 La législation scolaire française des années 1880 est inspirée par l'idée de libérer l'enseignement de l'influence des religieux. En effet, c'est la loi Goblet du 30 octobre 1886 sur l'organisation de l'enseignement primaire qui les en écarte, en ordonnant la laïcisation progressive du personnel enseignant des écoles primaires publiques ${ }^{6}$. En fin de compte, on peut dire que le 7 juillet 1904, les congrégations religieuses de France ont été supprimées. Au moins 30000 religieux et religieuses choisirent l'exil pour vivre comme ils en avaient fait le vœu (Prost 1968 : 54). Une partie d'entre-eux atteignit la Bulgarie.

7 Les écoles catholiques françaises en Bulgarie peuvent être identifiées au moment de leur ouverture comme des établissements privés (ou libres), fondés et entretenus par des associations (c'est-à-dire, de droit privé et non public), mais qui peuvent, malgré leur statut, recevoir des subventions, car elles sont reconnues d'utilité publique et présentent un intérêt national. Bien entendu, cette solution associe ces écoles au service public, en les soumettant à des obligations inspirées de ce dernier (Boussinesq 1994 : 111). Leur personnel enseignant doit être muni d'un brevet de capacité pour l'enseignement primaire. Et c'est le cas pour la plupart des enseignants des missions catholiques qui, en Bulgarie, suivent les programmes de l'enseignement primaire et des collèges en France, tout en respectant des conditions et des règles imposées par le milieu d'accueil.

8 Quant à la législation scolaire bulgare, la Loi sur l'instruction publique, du 10 décembre 1891, stipulait dans son art. 10, que les enfants des sujets Bulgares de religion chrétienne devraient recevoir leur instruction primaire en langue bulgare, et dans son art. 214, que les écoles étrangères qui recevaient des enfants de sujets bulgares devraient se conformer aux règlements des écoles nationales. Ces mesures, tout en visant plus particulièrement les écoles grecques, pouvaient avoir un contrecoup des plus fâcheux sur les écoles catholiques.

9 Le 13 janvier 1906, est signé le Traité de commerce et de navigation conclu entre la France et la Bulgarie. Selon le texte du traité, les établissements religieux et scolaires 
catholiques, existant en Bulgarie, seront dispensés des impôts, sauf pour les immeubles de rapport et pour les maisons où des élèves seraient admis en pension sans y recevoir d'instruction scolaire. Les subventions envoyées aux établissements catholiques par leurs instituts et les contributions qui leur sont payées par leurs élèves ne seront soumises à aucun impôt, en tant que lesdites subventions et rétributions sont affectées à l'entretien général des établissements ${ }^{7}$. La Loi sur l'instruction publique bulgare du 5 mars 1909, dans son art. 359, stipule que " les écoles privées peuvent être de tous les degrés et de toute espèce et avoir un programme conforme avec celui de l'État ou différent ». Selon l'art. 360, «l'enseignement dans les écoles privées a lieu en langue bulgare ou autre » et que " la langue bulgare, l'histoire bulgare et la géographie sont obligatoires pour tous les élèves bulgares qui s'instruisent dans ces écoles ». Ainsi, on voit bien que l'État se porte garant de l'enseignement de la langue bulgare et d'autres éléments contribuant à former l'identité nationale, tout en stimulant l'enseignement $\mathrm{du} / \mathrm{en}$ français à tous les degrés.

\section{Situation de la langue française en Bulgarie}

À la suite de la guerre russo-turque de 1877-1878, la Bulgarie est divisée par le traité de Berlin en deux parties : Principauté de Bulgarie et Roumélie orientale. Pendant la durée du régime organisé par le Statut organique de la Roumélie orientale, le français conserva une situation toute spéciale dans une province où les trois idiomes locaux bulgare, grec et turque étaient placés sur un pied d'égalité et où «chacune de ces trois nationalités mettait, pour ainsi dire, son point d'honneur à ignorer la langue des deux autres $»^{8}$. Le français était donc la langue de médiatisation par excellence.

11 L'union de la Roumélie à la Principauté bulgare, accomplie en 1885, a profondément modifié, de ce point de vue, l'état antérieur des choses. L'élément bulgare qui représentait les sept dixièmes de la population rouméliote, avait voulu s'attribuer par cette révolution une entière prédominance. La langue bulgare est en effet devenue, depuis lors, la seule langue officielle. Malgré les changements politiques et les conséquences entraînées par le réaménagement administratif à la suite de l'unification du pays, la langue française, ainsi que les établissements scolaires des missions catholiques qui assuraient sa propagation ont prospéré.

Un témoignage puisé dans les archives diplomatiques françaises fournit des observations intéressantes :

Quoiqu'il en soit, le français est resté parmi les idiomes de l'Europe occidentale de beaucoup le plus répandu dans l'ancienne province autonome. Il est l'organe courant des colonies étrangères, des nationalités diverses mais d'origine principalement levantine qui détiennent presque tout le haut commerce. Parlée couramment par les étrangers, très répandue dans le monde commercial et dans les classes les plus cultivées de la population bulgare, la langue française a même été mise par nos missionnaires, et par les écoles de l'Alliance israélite à la portée des classes populaires... Les écoles fournissent au commerce local des employés très appréciés par leur connaissance des idiomes du pays et particulièrement de la langue française; des œuvres d'apprentissage donnent l'enseignement professionnel aux élèves sortant de ces écoles... Mais c'est encore à l'étude de la langue française ou à des leçons faites en français que sont consacrées plus de la moitié des heures de classe ${ }^{9}$.

On peut penser que les écoles doivent leur essor, la multiplication des effectifs et les relations bienveillantes établies avec les gouvernements bulgares vers la fin du XIX et 
au début du $\mathrm{XX}^{\mathrm{e}}$ siècle, à l'utilité de la langue française. Cette utilité à la fois économique, politique et socioculturelle est susceptible de relier la «législation laïque » de la France républicaine et les réformes visant la stabilisation des institutions monarchiques en Bulgarie par les efforts des missions catholiques françaises motivées à scolariser et à œuvrer pour la propagation du français.

\section{Les Frères des écoles chrétiennes et le modèle lasallien}

14 Les congrégations autorisées, peu nombreuses, parmi lesquelles les Frères des écoles chrétiennes, sont supprimées en France, malgré le fait que «leurs succès et leur zèle étaient nettement reconnus par les pouvoirs publics» (Alban 1970: 272-273). Le nombre des frères dans ce pays, en 1903, est de 10 665, tandis qu'en 1913, ils sont déjà au nombre de 6657 (Sauvage 2002: 5). À la date du $1^{\mathrm{er}}$ janvier 1904, l'Institut lasallien en France comprend: «Maisons - 1157; Frères - 10651; Elèves - 201521; Sujets en formation - Scolastiques - 665 ; Novices - 529 ; Petits novices - 1442. Il comprend aussi hors de France : Maisons - 1563 ; Frères - 15432 ; Elèves - 321333 » (Ibid : 268).

Rappelons que le modèle d'éducation dessiné par Jean-Baptiste de la Salle repose sur des règles claires et précises :

-le frère consacre à l'école tout son temps, il ne peut avoir en même temps une autre obligation ;

-l'étude, et, par conséquent, l'enseignement du latin est interdit ;

-la lecture commence par celle du français ;

-l'usage de la méthode simultanée qui repose sur la présence et le contact du maître avec tous les élèves en même temps et la généralisation de cette méthode par les maitres lasalliens qui pratiquent simultanément l'apprentissage de la lecture et de l'écriture ;

-les frères enseignants sont obligés de vivre en communautés de deux ou trois frères au moins ;

-les écoles sont gratuites.

$16 \mathrm{Au} \mathrm{XIX}{ }^{\mathrm{e}}$ siècle, les frères contribuent à l'enseignement des sciences et des langues vivantes. Ils semblent être même les initiateurs du baccalauréat moderne qui est créé en France en 1891. Compte tenu du fait que les méthodes pédagogiques et éducatives font le succès de leurs écoles, il n'est pas étonnant, qu'en 1880, les frères soient sollicités par le conseiller du gouvernement bulgare pour les finances, Monsieur Hocdé, pour ouvrir à Sofia une école de langue française; pour cette école il a alloué une somme considérable (Imbert-Stanislas 1979: 46) ${ }^{10}$.

17 L'école est inaugurée en 1881 par un père assomptionniste puis passe sous la direction des Frères des écoles chrétiennes en 1885 après une entente cordiale entre les deux supérieurs généraux (Ibid.: 1). Le matin du 3 octobre 1881, 53 élèves sont présents : 25 catholiques, 28 orthodoxes, bientôt s'y ajoutent 18 juifs et 2 protestants. La bénédiction de l'école a lieu le 15 octobre.

L'école fonctionne comme une école bilingue (français et allemand), dirigée par les frères des districts de Belgique et d'Autriche, avec des frères français comme auxiliaires, jusqu'en 1916. Depuis cette année, elle est dirigée par les frères du district 
de Constantinople, avec le français comme langue d'enseignement, selon le vœu du donateur, M. Hocdé, sans, bien sûr, « négliger les autres langues » (Ibid.).

L'activité du frère Florin-Anatole, directeur du collège de 1908 à 1915, mérite notre attention pour plusieurs raisons. D'abord, l'école lui doit les constructions de 1910, 1912 et 1914 qui ont mis l'école « au large " pour les classes et pour la pension, et même pour le musée scolaire plein de spécimens tant autrichiens que français. Il a su trouver des ressources pour effectuer tous les travaux malgré les guerres successives. Son travail attire l'admiration mais aussi le soutien de la reine bulgare Éléonore qui après avoir apprécié la bonne tenue des élèves au collège, intervient personnellement pour obtenir la cession d'un terrain au profit de l'école des frères. Ensuite, il a réussi à lancer avec l'aide de son adjoint le frère Étienne, la section commerciale du collège à Sofia (Michel 2004 : 18-20). En 1912, pendant les vacances, avec l'aide des pères assomptionnistes, le collège adopte le programme de l'école bulgare de commerce de la ville de Svichtov, sur le Danube. La durée des études est de quatre ans et l'organisation est maintenue jusqu'à la fermeture du collège, en 1948.

Après la Grande Guerre, l'école est relancée par des frères français « détachés » qui viennent à Sofia. Le premier frère soldat démobilisé à la demande du commandant de l'armée française d'occupation en Bulgarie et qui revient à Sofia est le frère Étienne. Grâce aux frères soldats, l'année scolaire 1918-1919 n'est pas perdue pour les enfants de Sofia. Le soldat Margot redevenu le frère Antoine enseigne, entre autres, la sténo et la dactylo au cours commercial. Selon les témoignages de l'époque, il est « le plus aimé et le plus estimé de tous » et " cela n'a jamais changé en ces 30 années » (Ibid.: 50) ${ }^{11}$. Le sergent Prat nous rend ses émotions à la veille de son départ de Sofia après six mois d'enseignement à l'école de la rue Pirot/Lavélé : « [...] je vois ces six mois passés à Sofia et mes chers élèves, auxquels je me suis attaché comme je l'aurais fait pour de petits Français. [...] je sens une mélancolie doublée d'un serrement de cœur très réel [...]» $(\text { Ibid. })^{12}$. En cette période trouble d'après-guerre d'instabilité politique et sociale, l'école est un lieu protégé de travail, d'échange, de compassion et de stabilité morale.

Le 8 août 1921, le ministère de l'Instruction publique bulgare reconnaît le diplôme commercial du collège qui est équivalent aux diplômes des écoles commerciales de l'État en Bulgarie. À la rentrée de 1920-1921 on compte: 106 catholiques, 265 orthodoxes, 3 musulmans et 135 israélites, au total : 509 élèves (Ibid. : 51). Les élèves affluent pour atteindre 677, en 1922: 104 catholiques, 348 orthodoxes, 219 israélites et 6 musulmans (Ibid. : 31 ).

Entre 1928 et 1935, un nouveau projet audacieux est en cours. En août 1925, le ministère de l'Instruction publique bulgare autorise les frères à transférer une partie de l'école de la rue de Pirot à Lozenetz et permet l'ouverture d'une section classique, en acceptant la mise en place d'un lycée catholique. En 1928, le nouveau directeur, le frère Imbert-Stanislas (1890-1981) arrive d'Izmir. Il reste à Sofia pendant neuf ans et assume la construction et l'aménagement du collège - un gros travail qui exige beaucoup de fonds et d'efforts, et qui a commencé avant son arrivée. Une mobilisation exceptionnelle de ressources matérielles, financières et humaines est en cours et les travaux se dérouleront dans les délais prévus. L'ouverture du collège Saints Cyrille et Méthode sur la colline de Lozenetz aura lieu en 1935. L'aventure de Lozenetz est une décision réussie qui arrive à retourner durablement la tendance à la baisse des effectifs. 


\section{La vocation, l'appel, le vœu ${ }^{13}$} à travers les siècles dans l'histoire de l'église catholique. L'importance de la question est hors de doute et elle a fait couler beaucoup d'encre. Les débats menés à ce propos sont souvent liés à une remise en valeur des filières d'éducation des candidats dans l'institution. L'accent est mis autant sur « la nécessité d'un discernement de l'intention droite et de l'idonéité $~^{14}$, que sur l'importance de préserver le lien entre vocation divine et vocation ecclésiastique (Sauvage 2001:26-27). L'appel par Dieu et les signes de cet appel dans l'homme font partie des questions essentielles qui donnent sens à ces débats, tout en stimulant les aspirations au renouveau spirituel au sein de l'Église (Ibid.). Compte tenu de l'évolution des pratiques d'éducation et de formation des candidats et de leur activité pédagogique par la suite, on peut poser que les critères de sélection mais aussi les principes et les valeurs de formation des frères contribuent aux succès de leurs écoles en Orient, et en particulier en Bulgarie. nécrologiques, passés par le petit noviciat et le noviciat de Saint-Maurice l'Exil (Isère) de Caluire (Lyon) et de Rhodes en Grèce.. Ces nobles et modestes institutions sont des pépinières de futurs missionnaires et enseignants qui ont choisi l'exil. Ceux-ci se sentent appelés à éclairer les intelligences, à former les caractères et les consciences des jeunes. Ils ajoutent à leurs fonctions de maitres, celles de linger et de cuisinier, de tailleur et de sacristain, parfois même d'infirmier et d'économe, et malgré la déficience $\mathrm{du}$ ravitaillement et les conséquences des guerres, ils sont capables de dire comme le frère Palladius : «J'ai été heureux dans ma vie d'éducateur, je me félicite d'avoir choisi la meilleure part et d'avoir persévéré. $»^{15}$

Le 5 août 1885, l'évêque de Grenoble approuve et bénit le projet de noviciat apostolique à Saint-Maurice l'Exil, destiné à former des sujets pour les écoles d'Orient. Les Frères des écoles chrétiennes quitteront la maison de Saint-Maurice l'Exil, le 30 juin 1969.

\section{La Pédagogie lasallienne à Sofia}

Dans toutes les classes il s'agit en premier lieu d'apprendre le français. Lisons, à ce propos, les témoignages du détaché sergent Bruno, le frère Charles: "Pas d'autre moyen que la méthode directe : montrer un objet ou son image, dire son nom, le faire redire 10, 20 fois, ajouter un article, placer un verbe, en arriver à une petite phrase ; l'écrire au tableau, reposer la question à 2, 3 enfants, rectifier, recommencer. » (ImbertStanislas $1979: 48$ ).

En termes de méthode d'enseignement, le récit du frère Charles suggère des solutions :

« Pour arriver à transmettre à tous une indication générale, j'usais d'un stratagème : il y avait dans la classe une quinzaine de ces juifs, venus de Castille au XVI siècle, qui parlent espagnol dans l'intimité de la famille. J'en appelais un et lui disais à l'oreille en espagnol, ce que je voulais faire savoir à tous. Il se tournait vers ses camarades et le leur disait en bulgare. Une nouvelle chance : les nombreux tableaux Delmas et autres qui étaient dans l'école depuis le frère Florin. Ils enrichirent beaucoup et rapidement le vocabulaire des élèves et soulagèrent les professeurs. » (Ibid.).

Documents pour l'histoire du français langue étrangère ou seconde, 66-67 | 2021 
28 La lecture, le calcul, l'écriture, les leçons de choses dans les petites classes de l'école primaire de la rue Pirot/rue Lavélé préparent les élèves pour les défis au collège. Il est notoire que l'enseignement de qualité assuré au collège Saints Cyrille et Méthode à Sofia donne de l'importance aux mathématiques et aux sciences. Le brillant professeur de mathématiques et surtout de sciences dans les classes terminales, le frère François Kapsdorfer (1913-1981) passe sa vie religieuse et enseignante en Bulgarie. Il enseigne le matin au collège des frères et l'après-midi à l'école des Sœurs de Saint Joseph de l'Apparition ${ }^{16}$. Il est l'incarnation de l'idée du cardinal Saliège : «Un Frère des Écoles chrétiennes ne connaît pas de repos. Il travaille jusqu'au bout. C'est un exemple perpétuel de conscience professionnelle ${ }^{17}$. " C'est aussi un bel exemple d'élève qui dépasse son professeur. Son ancien maître de Saint-Maurice l'Exil se souvient: "J'expliquais un problème type au tableau noir et, ensuite, je donnais à faire 5 ou 6 exercices. François qui avait la bosse des maths, les résolvait tous et rapidement, sans erreurs et, dès le travail terminé, venait me présenter les résultats ${ }^{18}$. "

Les promenades et les randonnées organisées pendant les vacances par les frères, tout en étant un moyen de se reposer, favorisent la connaissance du pays où ils enseignent et permettent de mieux s'y attacher. Ils essayent d'oublier leur qualité d'étrangers, apprennent le bulgare, visitent les hauts lieux nationaux comme le monastère orthodoxe de Saint Jean et sa tombe dans le massif du Rila, les mines souterraines ou à ciel ouvert qui fournissent le charbon, l'usine de ciment "Granitoït», la plus importante en ce moment dans les Balkans etc., convaincus que ces visites favorisent leur attachement au pays.

Le frère Palladius (1872-1948) vient en Bulgarie, à Roussé, l'ancien Roustchouk sur le Danube, en 1928. Il y reste cinq ans. Lorsque les frères quittent cette ville, les supérieurs le destinent à Sofia, où le collège va bénéficier pendant quinze ans de son dévouement infatigable dans la section commerciale ou dans les classes dites spéciales. Dans sa classe règne une discipline parfaite, ce qui facilite singulièrement le travail. Il excelle à initier les élèves à la lecture, à la grammaire, à la conjugaison des verbes. Pour rendre ses leçons concrètes et vivantes, il emploie ses vacances à composer des tableaux d'enseignement collectif dont la belle disposition et la calligraphie charment les yeux et rendent les progrès rapides. Lors des compositions entre classes parallèles, ses élèves sont presque toujours vainqueurs. Les anciens surtout apprécient ses hautes qualités pédagogiques soulignées par le gouvernement français qui le nomme, officier d'académie en 1934. En dehors de sa classe, il s'occupe de la fanfare du collège, et quelques mois avant sa mort, il entraîne encore tambours et clairons dans les parades des fêtes religieuses. Aux fêtes de famille, lui-même joue volontiers, pour la grande joie de la communauté, quelques morceaux de son répertoire, un air de flûte ou de clarinette $^{19}$.

31 Le frère Cassin-Désiré (1877-1942), décoré de la croix de guerre, arrive à Sofia en 1922 et se dévoue aux petits Bulgares pendant quatre ans. «Son dévouement, la minutieuse préparation de ses leçons, et sa constance dans la correction des devoirs assuraient des progrès satisfaisants. ${ }^{20} \mathrm{Il}$ ajoute à ses fonctions de maître, celle de linger et de cuisinier - un travail supplémentaire considérable. «La confession régulière et la communion quotidienne complétaient les sources d'énergie spirituelle dont il sustentait son âme. $»^{21}$. les collèges de Smyrne et de Rhodes comme une grande partie des enseignants au 
collège de Sofia. Il ne châtie jamais, persuadé que l'exemple est le meilleur de tous les discours. En 1933, le gouvernement de la République française lui décerne les palmes académiques et deux ans plus tard, le tsar des Bulgares, Boris III, le fait chevalier du Mérite civil, pour son dévouement à la jeunesse bulgare ${ }^{22}$.

Le travail sans répit entre les murs des écoles des frères est soutenu par la bobine qui circule pendant les récréations pour réduire la communication en langue bulgare ou en toute autre langue entre les élèves en dehors des cours, par les billets hebdomadaires en témoignage de satisfaction pour motiver les élèves à travailler, récompenser leurs efforts et le travail effectué. Il faut noter aussi l'usage traditionnel des "bons points » dont la valeur varie selon la couleur, qui sont distribués aux élèves par les frères. Les notes hebdomadaires sont remises par le frère directeur dans chaque classe le samedi, accompagnées de notes de conduite et de travail. En 1928-1929, une nouveauté se fait remarquer : « un Palmarès avec la photo de toutes les classes, des élèves diplômés, des "Prix d'honneur" dans chaque classe, des groupements principaux dans l'école, fut remis à chacun des 717 élèves présents de la promotion. Les deux premiers élèves de chacune des 19 classes reçurent aussi un beau volume illustré, fourni et distribué par le Ministre de France » (Imbert-Stanislas 1979 : 66).

Le frère Pierre-Marie prend la direction du collège en 1936. En tant que directeur, il doit assumer le réquisitionnement du collège par les Russes pour en faire un hôpital, puis leur quartier général jusqu'en 1947. Le retour des frères est de courte durée car l'année suivante les écoles étrangères sont fermées et nationalisées avec la prise du pouvoir par les communistes. Le départ des frères devient alors inévitable et imminent. Il se fait sans panique sous la direction du frère Pierre-Marie qui reste sur place jusqu'en avril 1949 (Ibid. : 59).

\section{Conclusion}

Il sera utile de rappeler, en accord avec Jacques Thobie (2007 : § 27), « que la langue est un vecteur de culture et de civilisation" qui permet d'articuler des éléments de motivation différents et leur effet de synergie. Les effets du fonctionnement des écoles des frères et des écoles catholiques françaises en général au sein de la société bulgare semblent dépasser l'apprentissage de la langue française et la simple multiplication des Bulgares maîtrisant le français. Le statut de pays francophone, membre de l'OIF, n'est que la partie visible de ces effets.

Parmi les facteurs qui ont contribué au succès des trois écoles des Frères des écoles chrétiennes, il faut mentionner en premier la qualité de l'enseignement assurée, grâce à la motivation et la qualification professionnelle des frères. En accord avec SanchezSummerer (2015:33), l'étude de cas est susceptible de nous sensibiliser à la qualité et à l'efficacité des efforts individuels dans l'enseignement du français langue étrangère au croisement de stratégies et d'ambitions politiques et religieuses différentes.

Il semble important de noter, ensuite, l'engagement politique, le soutien sans faille apporté par la République française et plus précisément la souplesse et l'efficacité de l'action de ses agents diplomatiques. Notons aussi le soutien et l'aide de la monarchie bulgare $^{23}$, même s'ils sont inconséquents et de circonstance, en fonction d'orientations et de choix politiques complexes. 


\section{BIBLIOGRAPHIE}

\section{Sources primaires}

Archives lasalliennes, № 634 : État nominatif et statistique au 31 décembre 1939 et au 31 décembre 1940, Maison de Sofia, J. M. J. s.-j.-b., Institut des Frères des Écoles chrétiennes, district de Constantinople, collège français Saints Cyrille et Méthode - Sofia - Lozenetz.

ALBAN, FSC ${ }^{25}$ (1970). Histoire de l'Institut des Frères des Écoles Chrétiennes, Expansion HORS de France (1700-1966). Rome : Editions Générales F.S.C.

BÉDEL, Henri, FSC (2007). Initiation à l'histoire de l'Institut des Frères des Écoles chrétiennes. « XX ${ }^{\mathrm{e}}$ siècle 1928 - 1946 ». Études Lasalliennes 12. En ligne : https://www.lasalle.org/wp-content/uploads/ 2019/09/Études-Lasalliennes-12.pdf

BÉDEL, Henri, FSC (2004). Initiation à l'histoire de l'Institut des Frères des Écoles chrétiennes " $\mathrm{XIX}^{\mathrm{e}}-\mathrm{XX}^{\mathrm{e}}$ S. 1875 - 1928 ». Études Lasalliennes 11. En ligne : https://www.lasalle.org/wp-content/uploads/ 2019/09/\%C3\%89tudes-Lasalliennes-11.pdf IMBERT-STANISLAS, FSC (1979 [1980 ?]). Histoire de l'école des Frères de Sofia, Bulgarie. MICHEL, Ange, FSC (2004). Sofia : Le collège des SS. Cyrille et Méthode dirigé par Les Frères des Écoles chrétiennes. Amicale des anciens élèves des Frères.

SAUVAGE, Michel, FSC (2002). «La vie religieuse : esprit et structure ». Études Lasalliennes 10. Frères des Écoles chrétiennes, Maison Saint Jean-Baptiste de La Salle - 476, Via Aurelia, Rome.

SAUVAGE, Michel, FSC (2001). «Vie religieuse laïque et vocation de frère ». Études Lasalliennes 8. Frères des Écoles chrétiennes, Maison Saint Jean-Baptiste de La Salle - 476, Via Aurelia, Rome.

\section{Sources secondaires}

Boussinesq, Jean (1994). La laïcité française. Mémento juridique. Paris : Éditions du Seuil. IMBERT, Paul (1909). La rénovation de l'Empire ottoman. Affaires de Turquie. Paris : Perrin. 
PROST, Antoine (1968). Histoire de l'enseignement en France 1800-1967, Paris, Armand Colin.

ROCHE, Max (1989). Education, assistance et culture française dans l'Empire ottoman, 1784-1868. Istanbul : Isis.

SANCHEZ-SUMMERER, Karène (2015). «Pour Dieu et la Patrie. Formation des missionnaires français envoyés au Levant (1880-1940). Le cas des frères des écoles chrétiennes ». Documents pour l'histoire $d u$ français langue étrangère ou seconde, 55, 83-99. En ligne $:$ https://journals.openedition.org/ dhfles/4286

THOBIE, Jacques (2007). «L'importance des écoles dans la diffusion du français dans l'Empire ottoman au début du XXe siècle », Documents pour l'Histoire du Français Langue Étrangère ou Seconde, 38/39, 67-85. En ligne : https://journals.openedition.org/dhfles/140

zAïmova, Raïa (2007). « Le français en Bulgarie dans le contexte de la politique culturelle de la France aux XIX ${ }^{e}$ et XX $\mathrm{X}^{\mathrm{e}}$ siècles ». Documents pour l'Histoire du Français Langue Étrangère ou Seconde, 38/39, 149-156. En ligne : https://journals.openedition.org/dhfles/286

\section{NOTES}

1. Cette recherche a été possible grâce à l'aide précieuse du frère Alain Houry et l'accueil exceptionnel de la part de la communauté des frères des écoles chrétiennes et le service des archives lasalliennes de Lyon, en 2019. Je saisis cette occasion pour les remercier, ainsi que Madame Magali Devif, archiviste, responsable du service, et leur exprimer ma profonde reconnaissance et mon respect.

2. Selon la formule du frère Ange Michel.

3. L'école est ouverte en 1895.

4. The first Roman Catholic congregation of male non clerics devoted solely to schools, learning, and teaching.

5. La ville s'appelait Roustchouk jusqu'à la fin de la domination ottomane, en 1878.

6. Cf. Art. 17.- «Dans les écoles publiques de tout ordre, l'enseignement est exclusivement confié à un personnel laïque. » Loi du 30 octobre 1886 sur l'organisation de l'enseignement primaire. Journal officiel du 31 octobre 1886.

7. Lettre de S. Paprikoff, ministère des Affaires étrangères et des cultes, du 15 avril 1910, à S. Exc. M. Paléologue, envoyé extraordinaire et ministre plénipotentiaire de France, CADN, Sofia Ambassade 644PO/1 (137), carton 18.

8. MAE, Archives diplomatique, Série C Administratives 1876-1907, C-35, Alliance française, Divers, 135 : Envoi d'une note sur l'enseignement de la langue française, le 12 septembre 1893, du Consulat de France à Philippopolis à Son Excellence, Monsieur Develle, Ministre des Affaires étrangères, Paris.

9. Ibid.

10. Monsieur Hocdé a laissé 40000 francs au Père Timothée pour l'achat du terrain et des maisons.

11. D'après les notes du Sergent Prat.

12. Notes écrites le 25 juin 1919.

13. «Que les Frères soient admis dans ledit Institut dans la seizième ou dix-septième année de leur âge, qu'ils s'y engagent d'abord par des vœux de trois ans seulement, et qu'ils renouvellent ces vœux chaque année, jusqu'à ce qu'ils aient atteint et accompli leur vingt-cinquième année, auquel âge ils seront amenés à prononcer leurs vœux perpétuels». Texte de la Bulle In apostolicae dignitatis solio qui a figuré en tête de toutes les éditions des Règles de la première - imprimée en 1726 - à celle de 1947. (Sauvage $2002: 10$ ). 
14. Mot du droit canon. «L'idonéité, c'est l'existence des aptitudes nécessaires à la profession de l'état de vie ou à l'exercice du ministère auquel on serait appelé par Dieu ». (Sauvage 2001 : 29).

15. Cf. Institut des Frères des Écoles Chrétiennes (1948). Notices nécrologiques trimestrielles, janvier, février, mars, n²17 : 100-101. Paris.

16. Cf. (1983). Nos défunts. Recueil des notices des défunts de 1981 - Région France, 1-3.

17. Cf. Institut des Frères des Écoles Chrétiennes (1948). Notices nécrologiques trimestrielles, janvier, février, mars, $\mathrm{n}^{\circ} 217: 112$.

18. Témoignage du frère Yves Alancon, enseignant de mathématiques au Petit-Noviciat de SaintMaurice l'Exil (Isère) dans : (1983). Nos défunts. Recueil des notices des défunts de 1981 - Région France, 1.

19. Cf. Institut des Frères des Écoles Chrétiennes (1948). Notices nécrologiques trimestrielles, janvier, février, mars, $n^{\circ} 217: 98-102$.

20. Cf. Institut des Frères des Écoles Chrétiennes (1942). Notices nécrologiques, juillet, août, septembre, $\mathrm{n}^{\circ} 189: 4$.

21. Ibid.: 6 .

22. Cf. Institut des Frères des Écoles Chrétiennes (1943). Notices nécrologiques, nº191: 125.

23. La maison de Saxe-Cobourg et Gotha.

24. Par exemple, sur le diplôme (certificat de maturité) du collège français Saint Augustin à Plovdiv (anc. Philippopoli) de mon père Anguel Dimtchev, datant du 14 juin 1948, est écrit en haut, à droite: "Établissement reconnu par les gouvernements de France (janvier 1897), de Bulgarie (juin 1897), d'Autriche (mai 1912), de Hongrie (septembre 1912).

25. du latin Fratres Scholarum Christianarum.

\section{RÉSUMÉS}

Entre 1885 et 1948, plus d'une soixantaine de Frères des écoles chrétiennes, appartenant à " une congrégation originale $»^{2}$ fondée par Jean-Baptiste de La Salle en 1680, congrégation laïque masculine de droit pontifical, consacrent tout leur temps à leurs trois écoles lasalliennes en Bulgarie, dont deux à Sofia et une à Roussé ${ }^{3}$ (Roustchouk). Le collège Saints Cyrille et Méthode (1919-1948) est dénommé d'abord, selon un devis de 1926, «Collège Saint Jean-Baptiste de La Salle, à Sofia » du nom du fondateur des frères. L'excellente réputation de ces écoles attire les élèves qui forment "un ensemble très cosmopolite " et vivent en parfaite harmonie (Michel 2004 : 31). La langue française en est l'outil d'enseignement. La méthode simultanée contribue à la construction « d'une véritable communauté éducative », grâce au dévouement des frères.

Between 1885 and 1948, more than sixty Brothers of the Christian schools, belonging to "an original congregation" ${ }^{4}$ founded by Jean-Baptiste de La Salle in 1680, a lay male congregation of pontifical right, devoted all their time to their three Lasallian schools in Bulgaria, including two in Sofia and one in Ruse (Roustchouk). The college Saints Cyril and Methodius (1919-1948) is first called, according to a 1926 estimate, "College Saint Jean-Baptiste de La Salle, in Sofia" after the name of the founder of the Brothers. The excellent reputation of these schools attracts students who form "a very cosmopolitan whole" and live in perfect harmony. The French language is the teaching tool. The simultaneous method contributes to the construction of "a true educational community", thanks to the dedication of the brothers. 
INDEX

Mots-clés : Bulgarie, Frères des écoles chrétiennes, vocation, vœu, réseau lasallien

Keywords : Bulgaria, Brothers of the Christian schools, vocation, vow, Lasallian network

\section{AUTEUR}

\section{JULIETA VELICHKOVA-BORIN}

Université de Sofia Saint Clément d'Ohrid

julietaborin@yahoo.fr 